\title{
Endoplasmic reticulum stress and renal lesion in mice with combination of high-fat diet and streptozotocin-induced diabetes ${ }^{1}$
}

\author{
Hong Xie', Liu Huang ${ }^{\mathrm{II}}$, Yayun Li"I, Han Zhang ${ }^{\mathrm{IV}}$, Hao Liu ${ }^{\mathrm{V}}$ \\ DOI: http://dx.doi.org/10.1590/S0102-865020160030000001 \\ IAssociate Professor, Faculty of Nursing, Bengbu Medical College, Bengbu, Anhui, China. Intellectual and scientific content of the study, conception \\ and design of the study, manuscript writing.

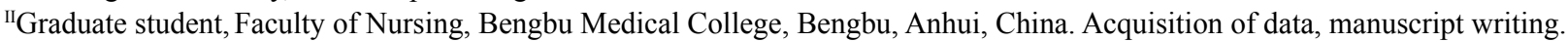

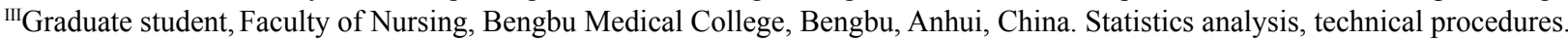 \\ ${ }^{\mathrm{IV}}$ Graduate student, Faculty of Nursing, Bengbu Medical College, Bengbu, Anhui, China. Analysis and interpretation of data. \\ ${ }^{v}$ Full Professor, Faculty of Pharmacy, Bengbu Medical College, Bengbu, Anhui, China. Critical revision, final approval.
}

\section{ABSTRACT}

PURPOSE: To investigate in the kidney the pathologic changes and expression of GRP78 and CHOP in the Kunming (KM) mice with combination of high-fat diet and streptozotocin-induced diabetes.

METHODS: Sixty two male KM mice were randomly divided into a normal control (NC) group (n=20) and a high-fat diet (HFD) group $(n=42)$. After a four-week dietary manipulation, the KM mice in the HFD group were injected intraperitoneally with streptozotocin to induce diabetes. After diabetic models were successfully established, the kidneys were excised and conserved for further test.

RESULTS: No significant difference in the body weight was observed after the dietary manipulation ( $p=0.554)$. After the streptozotocin was injected, fasting blood glucose levels in the diabetes group (DM) were significantly higher than that in the $\mathrm{NC}$ group ( $<<0.0001$ ). Glomerular atrophy observed under light microscope in the DM group was more serious compared with the NC group. The expression of GRP78 and CHOP in the kidneys of the mice in the DM group were higher compared with the NC group.

CONCLUSION: Renal lesion occurs in the diabetic Kunming mice induced by combination of high-fat diet and low-dose streptozotocin, and endoplasmic reticulum stress and CHOP may contribute to the injury process.

Key words: Endoplasmic Reticulum Stress. Transcription Factor CHOP. Diabetes Mellitus. Streptozocin. Mice. 


\section{Introduction}

During the past 30 years, the morbidity of diabetes mellitus (DM) in both China and the world increased steeply ${ }^{1}$. In 1980 , the morbidity of DM in China was only $0.67 \%$ according to a nationwide survey ${ }^{2}$, however in 2008 , the morbidity rose to $9.7 \% \%^{3}$. In 2013 , it even increased to $11.6 \%$, followed by a number of 113.9 million diabetics estimated in China according to the latest research ${ }^{4}$. Besides the high prevalence of DM, the morbidity of diabetic complications in China also rose due to the relatively short course of DM and the poorly controlled blood glucose. Of the complications, diabetic nephropathy (DN) stood out because of the aging population. Instead of glomerular disease, the diabetes and hypertension has become the leading cause of chronic kidney disease (CKD) in elder population in China ${ }^{5}$. As a result, the diabetic nephropathy will become the great challenge for the Chinese public medical service in the near future.

There are several theories including the inflammatory theory ${ }^{6}$, the oxidative stress theory ${ }^{7}$, and the Advanced Glycation End Products (AGEs) theory ${ }^{8}$, which try to explain the diabetes and the organ injuries during the diabetic complications. Recently endoplasmic reticulum (ER) stress received much attention. ER is an important organelle, which mainly responses for the synthesis and modification of protein, and ER stress is characterized by accumulated unfolded and misfolded protein in ER lumen. The accumulated protein interferes with the ER function, and activates the ER stress response, which is also called the unfolded protein response (UPR). UPR is intended to restore the homeostasis of ER and its function, whereas prolonged and excessive UPR plays an opposite role, which results in apoptosis and tissue injuries.

To our current knowledge, immunoglobulin heavy chain binding protein (Bip, also called GRP78) and C/EBP homologous protein (CHOP) are two important markers in the UPR. The GRP78, which helps to keep the homeostasis of ER, facilitates the folding of protein and GRP78 is overexpressed during the UPR. Nevertheless the upregulated CHOP induces apoptosis and tissue injuries during the UPR. Type 2 diabetes mellitus (T2DM) is characterized by insulin resistance and impaired insulin secretion. As pancreatic $\beta$-cell is the sole source of insulin, pancreatic $\beta$-cell plays a crucial role in the T2DM. Because pancreatic $\beta$-cells possess a highly-developed ER system, these cells are sensitive to the alteration of ER during the process of T2DM. Accumulating evidence has revealed that ER stress contributed to insulin resistance, aggravated $\beta$-cell burden, and reduced $\beta$-cell mass, resulting in $\mathrm{T}_{2} \mathrm{DM}^{9}$. Though two recent studies have proved that ER stress is activated in the kidney of diabetic rats ${ }^{10,11}$, whether
ER stress influences renal tissue in Kunming (KM) mice treated with high-fat diet and streptozotocin (STZ) is poorly understood. In this study, we aim to test whether renal lesion occurs in the diabetic Kunming mice induced by combination of high-fat diet and STZ. In addition, we also intend to evaluate whether ER stress is initiated in renal tissues.

\section{Methods}

The animal experimental protocols were approved by the Animal Experiments Ethics Committee of Bengbu medical college, and complied with the Guide for Care and Use of Laboratory Animals of Bengbu medical college.

Male KM mice aged four weeks were purchased from animal research center of Bengbu medical college. After one week of acclimatization period, they were randomly divided into a normal control group ( $\mathrm{NC}, \mathrm{n}=20$ ) fed with chow-diet consisting mainly (as a percentage of total weight) of $55 \%$ carbohydrate, $4 \%$ fat, $22 \%$ protein, $7 \%$ water, and $5 \%$ vitamins, and a highfat diet group (HFD, $\mathrm{n}=42$ ) fed with high-fat diet consisting mainly of $60 \%$ chow-diet, $10 \%$ lard, $10 \%$ yolk powder, and $20 \%$ sucrose. After a four-week dietary manipulation, all the mice were fasting for $12 \mathrm{~h}$. Afterwards, the mice in the high-fat diet group were injected intraperitoneally with $200 \mathrm{mg} \cdot \mathrm{kg}^{-1}$ body weight of streptozotocin (STZ, Sigma, St Louis, Mo.) dissolved in $0.1 \mathrm{M}$ citrate buffer $(\mathrm{pH}=4.4)$. Meanwhile, the mice in the normal control group were given equal volume of vehicle citrate buffer. A week after the STZ was injected, fasting blood glucose (FBG) levels were measured. Mice with a $\mathrm{FBG}>11.1 \mathrm{mmol} \cdot \mathrm{L}^{-1}$ were regarded as diabetic mice. Twelve mice in the HFD group were successfully made diabetes mellitus (DM group). After another week of dietary manipulation, mice were all sacrificed by decapitation and bilateral kidneys were excised. The kidneys used were stored in $4 \%$ paraformaldehyde solution for hematoxylin and eosin (HE) staining and immunohistochemical staining.

\section{Measurement of diet consumption and body weight}

During the 4-week dietary manipulation, diet was renewed and its consumption was calculated at 4 p.m. daily. Of the diet, the weight of fodder was measured by an electronic scale (Jingtian, Shanghai, China), and the volume of drinking water was measured by a graduated flask (Chilun, Shanghai, China). The daily fodder consumption was calculated by the difference of the weight of fodder renewed a day and the fodder remained the next day. The water consumption was calculated likewise. Then the mean of weekly diet consumption was calculated by the sum of 
daily diet consumption in a week divided by the number of mice in each group. The body weight (BW) was evaluated by an electronic scale (Jingtian, Shanghai, China) at the end of every week, and the average body weight of each group was calculated.

\section{Assessment of fasting blood glucose}

After the mice were fasting for $12 \mathrm{~h}$, the whole venous blood was isolated from the tail vein. The fasting blood glucose (FBG) levels were evaluated by a glucose meter (Yijie QS-B, Xiamen, China) with commercially available matched kits.

\section{Histopathology and immunohistochemical staining}

Renal samples were fixed in $4 \%$ paraformaldehyde solution, and embedded in paraffin blocks. Histologic sections were stained conventionally with hematoxylin-eosin $(\mathrm{HE})^{12}$ or for immunohistochemical staining ${ }^{13}$. The expression of GRP78 and $\mathrm{CHOP}$ in the renal tissue was respectively evaluated immunohistochemically with mouse polyclonal anti-GRP78 antibody (diluted 1:150, Santa Cruz Biotechnology, CA, USA) and rabbit polyclonal anti-CHOP antibody (diluted 1:200, Santa Cruz Biotechnology, CA, USA). The slides were observed under $\times 400$ magnification.

\section{Statistical analysis}

Data are presented as the means \pm SD. Statistical comparison between the two groups were evaluated by the
Student's t-test using SPSS 22.0 software (IBM Corp., Chicago IL). Statistical significance was set at $\mathrm{p}<0.05$.

\section{Results}

\section{Diet consumption and body weight alteration}

During the 4-week dietary manipulation, the average diet consumption of the NC group rose significantly between the first two weeks, and the diet consumption of the HFD group presented no obvious alterations. On average, the mice in the NC group consumed more chow-diet by weight, compared with the high-fat diet consumed by the mice in the HFD group every week (Figure 1A). The water consumption decreased between the week one and the week five. The mice in the NC group consumed more water than those in the HFD group did, however the difference was not statistically significant (Figure 1B). Body weights of the both groups increased with age. In addition, the body weights between the two groups revealed no significant difference (Figure 1C).

\section{Development of overt diabetes}

A week five after STZ was injected, the FBG of the mice in the high-fat diet group significantly increased compared with the mice in the normal control group, and the mice with a $\mathrm{FBG}>11.1 \mathrm{mmol} / \mathrm{L}$ were regarded as diabetic mice, which composed the diabetes mellitus (DM) group (Figure 1D).
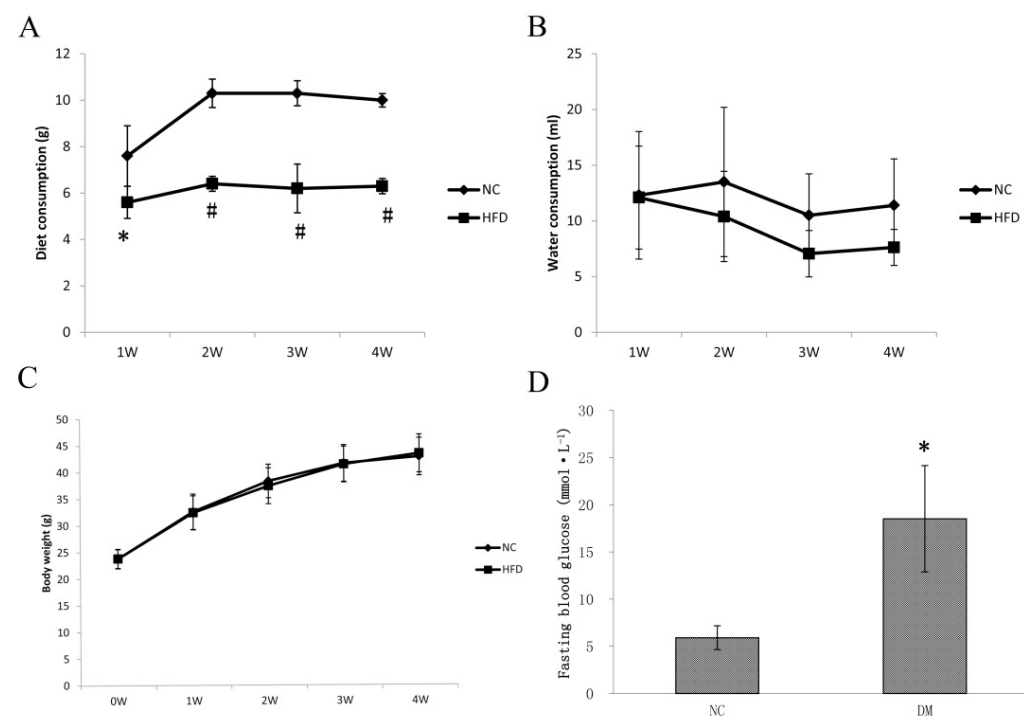

FIGURE 1 - (A) The diet of the normal control group (NC) and the high-fat diet group (HFD). (B) Water consumption of the normal control group (NC) and the high-fat diet group (HFD). (C) Variation of body weight in the normal control group (NC) and the high-fat diet group (HFD). (p=0.554) (D) The Fasting blood glucose after injected with streptozotocin in the diabetes mellitus group (DM). ${ }^{*} \mathrm{p}<0.05$ vs normal control (NC) group, \#p $<0.01$ vs NC group. 


\section{Pathological changes in renal lesion}

Glomerular atrophy, hyperaemia and thickness of the glomerular basement membrane as well as the tubular atrophy were observed in the renal samples of the mice in the DM group, while no apparent lesion was observed at the renal glomerulus and tubules in the samples of the mice in the NC group at week 6 (Figure 2A). It demonstrated that renal lesion occurred in the diabetic mice models.

\section{Expression of GRP78 and CHOP in the kidney}

GRP78 is a critical chaperone protein, which facilitates folding protein in ER. As a result, it is regarded as a key indicator for activated ER stress. Figure $2 \mathrm{~B}$ displayed the overexpression of GRP78 in kidney of mice in the DM group, compared with the weak immunoreactivity in $\mathrm{NC}$ group. $\mathrm{CHOP}$ is believed to result in apoptosis during the ER stress, so it is an important marker, which prompts the tissue injury in the ER stress. As showed in Figure 2C, CHOP expressed much more in the renal samples of the mice in the DM group compared with those in the NC group.
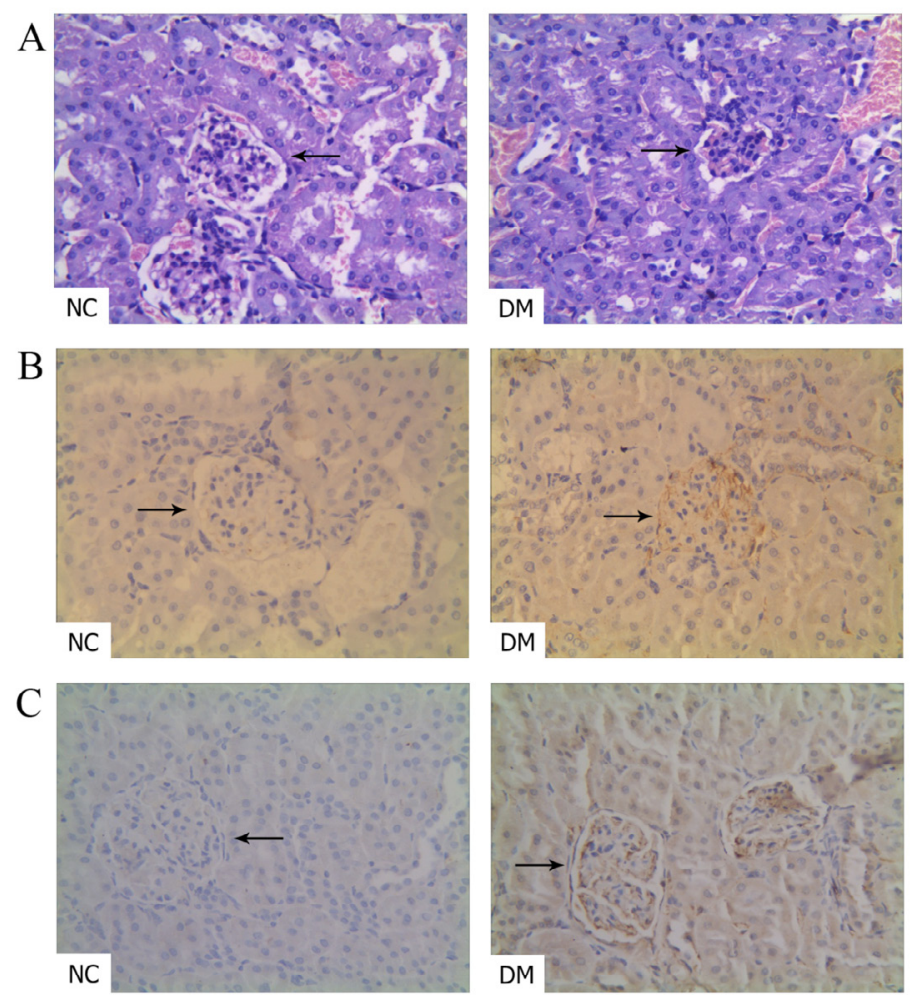

FIGURE 2 - (A) The light microscopy of renal histology by HE staining in the normal control group (NC) and the diabetes mellitus group (DM). (B) The expression of GRP78 in the kidney of the normal control group (NC) and the diabetes mellitus group (DM). (C) The expression of CHOP in the kidney of the normal control group (NC) and the diabetes mellitus group (DM). Original magnification: $\mathrm{x} 400$.

\section{Discussion}

There are several congenital diabetic models such as ob/ ob diabetic mice ${ }^{14}$, and other ways to establish acquired diabetes in rodent animals by alloxan ${ }^{15}$ and hydrocortisone ${ }^{16}$. However, with further research into DM, diabetes nephropathy (DN), one of the common complications of diabetes, received increasing attention. Therefore, rodent models which are able to mimic the process of the diabetic complications are crucial for study on DN. As STZ relatively specifically damages the pancreas islet, it becomes an ideal material to induce diabetes in rodent models. High-fat diet is able to cause insulin resistance (IR) in rodent models, and type 2 diabetes mellitus (T2DM) is characterized by IR, so the combination of high-fat diet and STZ are used to mimic the process of T2DM in animal models. Some study showed that the rats in high-fat diet group gained more body weight than those in the normal control group ${ }^{17}$, and in theory this makes sense. However, we did not observe this phenomenon that the mice in the HFD group are heavier than those in the NC group. This is partly because mice in the HFD group consumed less fodder by weight than those in the NC group as showed in Fig. 1A. As a result, the impact of the high-diet on other index such as serum cholesterol and triglyceride is well worth considering due to the consumption between the two types of diets. Usually the diabetic models were made from rats by combination of high-fat diet and low dose of $\mathrm{STZ}^{18}$, and the DN was usually induced by mono-nephrectomy based on the diabetic models ${ }^{19,20}$. Here we made a diabetic model with renal lesion out of the Kunming mouse, which is widely used for pharmacological and toxicological study in China. In addition, we also found the overexpression of ER stress indicators such as GRP78 and CHOP in renal tissue, and the obvious renal lesion in Kunming mice treated with high-fat diet and STZ. Though we successfully established a mice model with nephropathy and observed the upregulated GRP78 and CHOP in this model, we were not able to directly prove that ER stress did participate in the renal lesion of diabetes. Some study has proved that ER stress contributed to the renal injury in diabetic rats induced by STZ ${ }^{10,21,22}$, thus we conjecture that both models share the same mechanism on the onset of DN. We believe that this mechanism on DN can be explored by inhibiting the ER stress using an ER stress inhibitors such as 4-phenylbutyric acid (4-PBA). Besides, we believe that there are two directions for ER stress on DN research. One is to explore the relationship between the ER stress, oxidative stress and the metabolic inflammation, as we noticed the other two cellular responses also participate in the process of diabetes and 
complications, thus ER stress is just in part responsible for DM. The other is to focus on the pathways of ER stress. As GRP78 and CHOP plays different roles in the ER stress, and there are many other molecules such as inositol-requiring enzyme 1 (IRE1), PKR-like ER kinase (PERK), and activating transcription factor 6 (ATF6) involved in ER stress, the ER stress itself is a complex process. To understand the relationship between the ER stress and DN, we ought to comprehend the molecular pathway of ER stress first.

\section{Conclusion}

This study establishes a Kunming mice model of combination of high-fat diet and STZ-induced diabetes, and ER stress may contribute to the renal lesion in this diabetic model.

\section{References}

1. Finucane MM, Stevens GA, Cowan MJ, Danaei G, Lin JK, Paciorek CJ, Singh GM, Gutierrez HR, Lu Y, Bahalim AN, Farzadfar F, Riley LM, Ezzati M. National, regional, and global trends in body-mass index since 1980: systematic analysis of health examination surveys and epidemiological studies with 960 country-years and 9.1 million participants. Lancet. 2011 Feb 12;377(9765):557-67. doi: 10.1016/ s0140-6736(10)62037-5.

2. [A mass survey of diabetes mellitus in a population of 300,000 in 14 provinces and municipalities in China (author's transl)]. Zhonghua Nei Ke Za Zhi. 1981 Nov;20(11):678-83. PMID: 7341098.

3. Yang SH, Dou KF, Song WJ. Prevalence of diabetes among men and women in China. N Engl J Med. 2010 Jun 24;362(25):2425-6; author reply 6. PMID: 20578276.

4. Xu Y, Wang L, He J, Bi Y, Li M, Wang T, Wang L, Jiang Y, Dai M, Lu J, Xu M, Li Y, Hu N, Li J, Mi S, Chen CS, Li G, Mu Y, Zhao J, Kong L, Chen J, Lai S, Wang W, Zhao W, Ning G, China Noncommunicable Disease Surveillance G. Prevalence and control of diabetes in Chinese adults. JAMA. 2013 Sep 4;310(9):948-59. doi: 10.1001/jama.2013.168118.

5. Cao Y, Li W, Yang G, Liu Y, Li X. Diabetes and hypertension have become leading causes of CKD in Chinese elderly patients: a comparison between 1990-1991 and 2009-2010. Int Urol Nephrol. 2012 Aug;44(4):1269-76. doi: 10.1007/s11255-012-0194-0.

6. Chan JY, Biden TJ, Laybutt DR. Cross-talk between the unfolded protein response and nuclear factor-kappaB signalling pathways regulates cytokine-mediated beta cell death in MIN6 cells and isolated mouse islets. Diabetologia. 2012 Nov;55(11):2999-3009. doi: 10.1007/s00125-012-2657-3.

7. Wang X, Gu C, He W, Ye X, Chen H, Zhang X, Hai C. Glucose oxidase induces insulin resistance via influencing multiple targets in vitro and in vivo: the central role of oxidative stress. Biochimie. 2012 Aug;94(8):1705-17. doi: 10.1016/j.biochi.2012.03.024.

8. Schalkwijk CG, Miyata T. Early- and advanced non-enzymatic glycation in diabetic vascular complications: the search for therapeutics. Amino Acids. 2012 Apr;42(4):1193-204. doi: 10.1007/ s00726-010-0779-9.

9. Krokowski D, Han J, Saikia M, Majumder M, Yuan CL, Guan BJ, Bevilacqua E, Bussolati O, Broer S, Arvan P, Tchorzewski M, Snider
MD, Puchowicz M, Croniger CM, Kimball SR, Pan T, Koromilas AE, Kaufman RJ, Hatzoglou M. A self-defeating anabolic program leads to beta-cell apoptosis in endoplasmic reticulum stress-induced diabetes via regulation of amino acid flux. J Biol Chem. 2013 Jun 14;288(24):17202-13. doi: 10.1074/jbc.M113.466920.

10. Wang ZS, Xiong F, Xie XH, Chen D, Pan JH, Cheng L. Astragaloside IV attenuates proteinuria in streptozotocin-induced diabetic nephropathy via the inhibition of endoplasmic reticulum stress. BMC Nephrol. 2015;16:44. doi: 10.1186/s12882-015-0031-7.

11. Shao D, Liu J, Ni J, Wang Z, Shen Y, Zhou L, Huang Y, Wang J, Xue H, Zhang W, Lu L. Suppression of XBP1S mediates high glucose-induced oxidative stress and extracellular matrix synthesis in renal mesangial cell and kidney of diabetic rats. PLoS One. 2013;8(2):e56124. doi: 10.1371/journal.pone.0056124.

12. Ang L, Yuguang L, Liying W, Shuying Z, Liting X, Shumin W. Ergosterol alleviates kidney injury in streptozotocin-induced diabetic mice. Evid Based Complement Alternat Med. 2015;2015:691594. doi: 10.1155/2015/691594.

13. Kim H, Moon SY, Kim JS, Baek CH, Kim M, Min JY, Lee SK. Activation of AMP-activated protein kinase inhibits ER stress and renal fibrosis. Am J Physiol Renal Physiol. 2015 Feb 1;308(3):F22636. doi: 10.1152/ajprenal.00495.2014.

14. Ozcan U, Yilmaz E, Ozcan L, Furuhashi M, Vaillancourt E, Smith RO, Gorgun CZ, Hotamisligil GS. Chemical chaperones reduce ER stress and restore glucose homeostasis in a mouse model of type 2 diabetes. Science. 2006 Aug 25;313(5790):1137-40. doi: 10.1126/ science. 1128294.

15. Abd El Latif A, El Bialy Bel S, Mahboub HD, Abd Eldaim MA. Moringa oleifera leaf extract ameliorates alloxan-induced diabetes in rats by regeneration of beta cells and reduction of pyruvate carboxylase expression. Biochem Cell Biol. 2014 Oct;92(5):413-9. doi: 10.1139/bcb-2014-0081.

16. Xu TY, Chen RH, Wang P, Zhang RY, Ke SF, Miao CY. 4-Phenyl butyric acid does not generally reduce glucose levels in rodent models of diabetes. Clin Exp Pharmacol Physiol. 2010 Apr;37(4):441-6. doi: 10.1111/j.1440-1681.2009.05328.x.

17. Reed MJ, Meszaros K, Entes LJ, Claypool MD, Pinkett JG, Gadbois TM, Reaven GM. A new rat model of type 2 diabetes: the fat-fed, streptozotocin-treated rat. Metabolism. 2000 Nov;49(11):1390-4. doi: 10.1053/meta.2000.17721.

18. Srinivasan K, Viswanad B, Asrat L, Kaul CL, Ramarao P. Combination of high-fat diet-fed and low-dose streptozotocintreated rat: a model for type 2 diabetes and pharmacological screening. Pharmacol Res. 2005 Oct;52(4):313-20. doi: 10.1016/j. phrs.2005.05.004.

19. Sugano M, Yamato H, Hayashi T, Ochiai H, Kakuchi J, Goto S, Nishijima F, Iino N, Kazama JJ, Takeuchi T, Mokuda O, Ishikawa T, Okazaki R. High-fat diet in low-dose-streptozotocin-treated heminephrectomized rats induces all features of human type 2 diabetic nephropathy: a new rat model of diabetic nephropathy. Nutr Metab Cardiovasc Dis. 2006 Oct;16(7):477-84. doi: 10.1016/j. numecd.2005.08.007.

20. Luo ZF, Feng B, Mu J, Qi W, Zeng W, Guo YH, Pang Q, Ye ZL, Liu L, Yuan FH. Effects of 4-phenylbutyric acid on the process and development of diabetic nephropathy induced in rats by streptozotocin: regulation of endoplasmic reticulum stress-oxidative activation. Toxicol Appl Pharmacol. 2010 Jul;246(1-2):49-57. doi: 10.1016/j.taap.2010.04.005.

21. Qi W, Mu J, Luo ZF, Zeng W, Guo YH, Pang Q, Ye ZL, Liu L, Yuan $\mathrm{FH}$, Feng B. Attenuation of diabetic nephropathy in diabetes rats induced by streptozotocin by regulating the endoplasmic reticulum stress inflammatory response. Metabolism. 2011 May;60(5):594603. doi: 10.1016/j.metabol.2010.07.021. 
22. Liu G, Sun Y, Li Z, Song T, Wang H, Zhang Y, Ge Z. Apoptosis induced by endoplasmic reticulum stress involved in diabetic kidney disease. Biochem Biophys Res Commun. 2008 Jun 13;370(4):6516. doi: 10.1016/j.bbrc.2008.04.031.

\section{Acknowledgement}

To fellow schoolmates for their support during the research.

\section{Correspondence:}

Hao Liu

Faculty of Pharmacy, Bengbu Medical College, Bengbu, Anhui, China

Phone: + 865523175230

Fax: + 865523175228

liuhao6886@foxmail.com

Received: Nov 16, 2015

Review: Jan 17, 2016

Accepted: Feb 19, 2016

Conflict of interest: none

Financial source: National Natural Science Foundation of China (81000992), Creative Scientific Research Project for Postgraduate of Bengbu Medical College (Byycxz1408)

${ }^{1}$ Research performed at Faculty of Pharmacy, Bengbu Medical College, Bengbu, Anhui, China. 